\title{
Relationship between Atomic Structure, Composition, and Dielectric Constant in $\mathrm{Zr}-\mathrm{SiO}_{2}$ Glasses
}

\author{
S. Arash Sheikholeslam,* Jon López-Zorrilla,* Hegoi Manzano,* Saamaan Pourtavakoli,* \\ and André Ivanov*
}

Cite This: ACS Omega 2021, 6, 28561-28568

Read Online

ACCESS

山ll Metrics \& More

回 Article Recommendations

Supporting Information

ABSTRACT: Computational methods, or computer-aided material design (CAMD), used for the analysis and design of materials have a relatively long history. However, the applicability of CAMD has been limited by the scales of computational resources generally available in the past. The surge in computational power seen in recent years is enabling the applicability of CAMD to unprecedented levels. Here, we focus on the CAMD for materials critical for the continued advancement of the complementary metal oxide semiconductor (CMOS) semiconductor technology. In particular, we apply CAMD to the engineering of high-

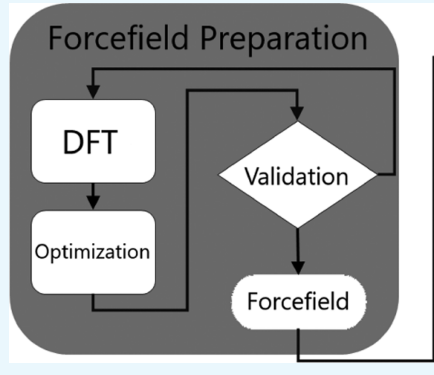
Forcefield Application permittivity dielectric materials. We developed a Reax forcefield that includes $\mathrm{Si}, \mathrm{O}, \mathrm{Zr}$, and $\mathrm{H}$. We used this forcefield in a series of simulations to compute the static dielectric constant of silica glasses for low $\mathrm{Zr}$ concentration using a classical molecular dynamics approach. Our results are compared against experimental values. Not only does our work reveal numerical estimations on $\mathrm{ZrO}_{2}$-doped silica dielectrics, it also provides a foundation and demonstration of how CAMD can enable the engineering of materials of critical importance for advanced CMOS technology nodes.

\section{INTRODUCTION}

Complementary metal oxide semiconductor (CMOS) device scaling has been driving the need for high relative permittivity (high- $k$ ) dielectrics. During fabrication, high- $k$ dielectrics are typically either directly deposited on top of the silicon channel (one layer oxide) or on an intermediate layer of $\mathrm{SiO}_{2}$ with a thickness of approximately $1 \mathrm{~nm} .{ }^{1}$ A dielectric material such as $\mathrm{Hf} / \mathrm{ZrO}_{2}$ was considered to replace the otherwise commonly used $\mathrm{SiO}_{2}{ }^{2}$. Of the two, $\mathrm{ZrO}_{2}$ found fewer applications in advanced CMOS technologies ${ }^{3-5}$ and is therefore less studied.

This article reports on our work that used molecular dynamics (MD)-based computer-aided material design (CAMD) methods to predict the dielectric constant of amorphous solids. In particular, we use classical $\mathrm{MD}$ to model bulk amorphous $\left(\mathrm{ZrO}_{2}\right)_{x}\left(\mathrm{SiO}_{2}\right)_{1-x}$, where the $\mathrm{ZrO}_{2}$ content can be tuned to achieve a certain desired dielectric constant. CAMD constitutes a set of alternative approaches for material discovery and characterization which can potentially reduce the associated material discovery costs via prescreening and redirecting the physical lab efforts. Different CAMD algorithms have been devised and used to date. These different algorithms are generally based on numerical simulations that, in turn, are based on different levels of abstractions of the physical material at hand. ${ }^{6,7}$ Among these different algorithms, those generally referred to as $a b$ initio methods are known to be the most accurate and have successfully been applied for material discovery purposes. Unfortunately, while $a b$ initio methods may be the most accurate, they tend to be computationally prohibitive for handling any "at scale" molecular system. Methods that have been devised to overcome the latter limitations by using simpler models include classical MD methods.

In our specific application of MD-based CAMD, we essentially adapted and customized an approach previously used in various contexts by other groups. ${ }^{8-12}$ This approach consists of parameterizing and optimizing classical forcefields that are subsequently used in MD simulations to determine particular characteristics of new or existing materials. Our CAMD algorithm can be broken down into two major procedural steps: a Force field Preparation step followed by a $M D$ simulation step. The force field preparation consists of the following:

(A) $A b$ initio simulations: Quantum chemistry methods are used to perform calculations on a set of molecular structures to characterize them in terms their ground state energy, structure, partial charges, and so forth.

Received: May 20, 2021

Accepted: October 12, 2021

Published: October 21, 2021 

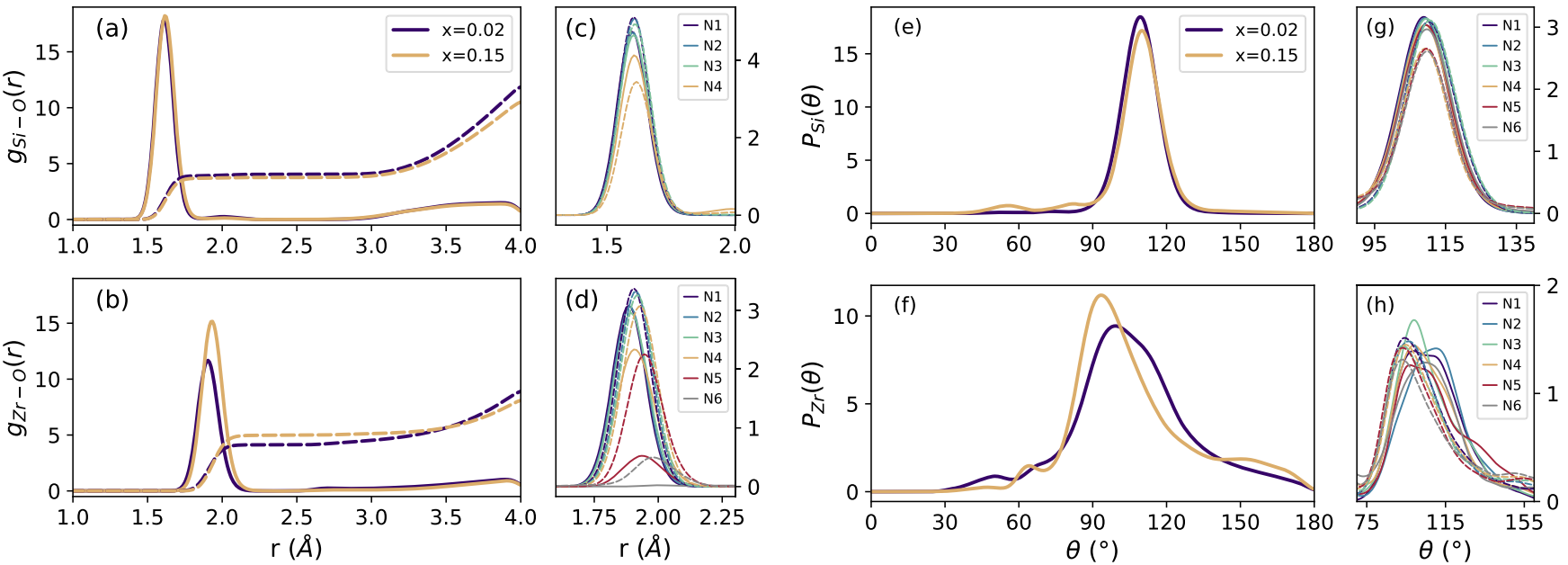

Figure 1. (a) $\mathrm{Si}-\mathrm{O}$ and (b) $\mathrm{Zr}-\mathrm{O}$ pair distribution functions for $x=0.02$ and $x=0.15$. Dashed lines show the coordination of each species as a function of the distance. The decomposition of the first peak of the distribution among the contributions of the nearest neighbors are shown on the right for both (c) $\mathrm{Si}-\mathrm{O}$ and (d) $\mathrm{Zr}-\mathrm{O}$ and (e) $\mathrm{Si}$ - and (f) $\mathrm{Zr}$-centered partial bond angle distributions. Solid lines are for $x=0.02$, and dashed lines are for 0.15 . The decomposition of the distributions among the contributions of the triangles formed by the nearest neighbors is shown on the right for both $(\mathrm{g}) \mathrm{O}-\mathrm{Si}-$ $\mathrm{O}$ and $(\mathrm{h}) \mathrm{O}-\mathrm{Zr}-\mathrm{O}$.

Table 1. General Structural Characteristics of $\left(\mathrm{ZrO}_{2}\right)_{x}\left(\mathrm{SiO}_{2}\right)_{1-x}$

\begin{tabular}{|c|c|c|c|c|c|c|c|c|}
\hline structure & $\mathrm{Si}-\mathrm{O}(\AA)$ & $\mathrm{Zr}-\mathrm{O}(\AA)$ & $\mathrm{O}-\mathrm{Si}-\mathrm{O}(\mathrm{deg})$ & $\mathrm{O}-\mathrm{Zr}-\mathrm{O}(\mathrm{deg})$ & $\mathrm{Si}-\mathrm{O}-\mathrm{Zr}(\mathrm{deg})$ & $\mathrm{Si}-\mathrm{O}-\mathrm{Si}(\mathrm{deg})$ & $\langle\mathrm{CN}\rangle$ & density $(\mathrm{g} / \mathrm{cm} 3)$ \\
\hline$x=0.00$ & 1.62 & & & & & & 3.98 & 2.20 \\
\hline$x=0.02$ & 1.63 & 1.88 & 109.09 & 108.44 & 142.23 & 137.69 & 4.08 & 2.25 \\
\hline$x=0.05$ & 1.63 & 1.89 & 109.19 & 107.47 & 142.16 & 136.65 & 4.36 & 2.26 \\
\hline$x=0.10$ & 1.63 & 1.90 & 109.53 & 106.09 & 141.52 & 135.68 & 4.72 & 2.30 \\
\hline$x=0.15$ & 1.63 & 1.90 & 110.11 & 105.68 & 142.58 & 134.52 & 4.92 & 2.30 \\
\hline$x=1.00$ & & 2.05 & & & & & 5.5 & 4.07 \\
\hline
\end{tabular}

(B) Optimization: A forcefield adequate for the material and application is chosen, and its relevant parameters are optimized. The parameter optimization is driven such that the MD simulations using the forcefield would accurately estimate the $a b$ initio results obtained in step (A). Some applications may warrant a validation of the optimized forcefield, particularly when the basis forcefield is a newly developed one.

One can then apply the resulting force field in MD simulations to discover or ascertain particular material characteristics. For this purpose, two MD simulations steps are necessary:

(C) Sample Material Development: MD is used at this step for virtual sample preparation. This sample preparation needs to give consideration to the physical context and boundary conditions of the material of interest.

(D) Material Characterization: This stage of the algorithm amounts to running $\mathrm{MD}$ simulations and performing computations on the simulation results to determine yet unknown characteristics of the material of interest.

The Methods section of this article presents a detailed description of the above CAMD algorithm in our work. We then present and discuss the particular dielectric constant results we generated for $\mathrm{Zr}$-doped silica glasses and the underlying atomic scale properties that govern it. A Supporting Information document complements this article. The latter provides more information and details on the following aspects of our work: forcefield description and parameters, forcefield optimization procedure and its implementation code, and dielectric constant computations.

\section{RESULTS AND DISCUSSION}

Structural Characterization. First, we analyze the atomic structure of $\left(\mathrm{ZrO}_{2}\right)_{x}\left(\mathrm{SiO}_{2}\right)_{1-x}$ glasses for various $\mathrm{x}$ values. In Figure 1 we show the radial distribution functions for $\mathrm{Si}-\mathrm{O}$ and $\mathrm{Zr}-\mathrm{O}$ pairs for the highest and lowest $\mathrm{Zr}$ contents. Both radial distribution functions present a sharp peak for the first neighbors without clear secondary peaks, a common characteristic of highly disordered materials. The $g_{\mathrm{Si}-\mathrm{O}}(r)$ is almost identical for both compositions, which indicates that the local tetrahedral arrangement of the silicate groups is not affected by the $\mathrm{Zr}$ content. On the other hand, there are significant changes in the $g_{\mathrm{Zr}-\mathrm{O}}(r)$ : the first peak is shifted to larger values (see Table 1 ) and the average coordination number $(\mathrm{CN})$ increases from $\approx 4$ to $\approx 5$. A more detailed analysis decomposing the first peak into partial contributions reveals that $\mathrm{Zr}$ is mainly 4-coordinated [ $\mathrm{Zr}(\mathrm{IV})]$ in the $0.02 \% \mathrm{Zr}$ sample and evolves to 4,5 -coordinated $[\mathrm{Zr}(\mathrm{IV})$ and $\mathrm{Zr}(\mathrm{V})]$ environments with a considerable 6coordinated $[\mathrm{Zr}(\mathrm{VI})]$ contribution. We also observed the presence of 3 -fold coordinated oxygen atoms, known as "tricluster" oxygen, similar to what has been reported for silicate and aluminosilicate glasses. ${ }^{13-15}$

The angular distribution functions for the $\mathrm{O}-\mathrm{Si}-\mathrm{O}$ and $\mathrm{O}-$ $\mathrm{Zr}-\mathrm{O}$ angles presented in Figure 1 also reflect the change in the coordination environments. The $\mathrm{O}-\mathrm{Si}-\mathrm{O}$ angles are centered at $109^{\circ}$, corresponding to the tetrahedral geometry, without significant differences for different $\mathrm{Zr}$ contents. In contrast, the $\mathrm{O}-\mathrm{Zr}-\mathrm{O}$ angle distribution is considerably wider, with a maximum value that shifts toward lower angles as the $\mathrm{Zr}$ content increases. That angle decrease is consistent with the evolution of some $\mathrm{Zr}(\mathrm{IV})$ sites from tetrahedral coordination to higher $\mathrm{CN}$ 
arrangements like the square pyramidal $\mathrm{Zr}(\mathrm{V})$ and octahedral $\mathrm{Zr}(\mathrm{VI})$.

We quantified in detail the $\mathrm{Zr}$-average $\mathrm{CN}$ as a function of the $\mathrm{Zr}$ content, as presented in Figure 2. We obtained an increasing

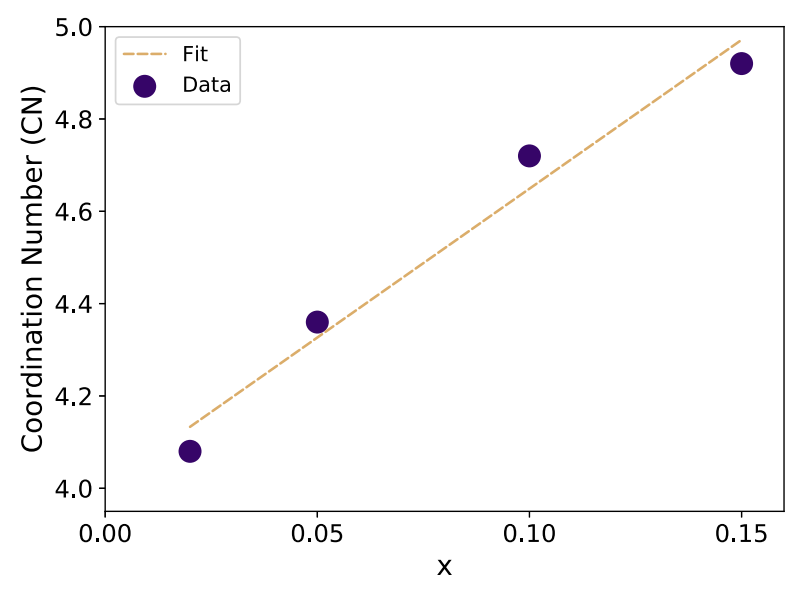

Figure 2. Average $\mathrm{ZrCN}$ as a function of $\mathrm{ZrO}_{2}$ content.

$\mathrm{Zr}$-average $\mathrm{CN}$ with the concentration of $\mathrm{Zr}$, consistent with the experimental observations using IR spectroscopy from Lucovsky and Rayner. ${ }^{16}$ As observable in Figure 2, we obtained a good linear fit $(\mathrm{CN}=a+b x)$ with the parameters $a \approx 4.00$ and $b \approx$ 6.42. The extrapolation to $x=0.5$ in the linear fit leads to $\langle\mathrm{CN}\rangle$ $\approx 7.2$ which is the expected average $\mathrm{CN}$ for $\mathrm{Zr}$ in amorphous zircon. $^{16,17}$

Other structural parameters are detailed in Table 1 and have been discussed earlier in the text.

Dielectric Constant. The main objective of this work is to compute the dielectric constants of $\left(\mathrm{ZrO}_{2}\right)_{x}\left(\mathrm{SiO}_{2}\right)_{1-x}$ from the dipole moment fluctuations. Before discussing the final values, it is important to pay attention to the convergence to a stable/ asymptotic value of the dielectric constant. As shown in Figure 3, these convergence rates varied for different compositions. The convergence was faster for pure $\mathrm{SiO}_{2}$ than those for the $\left(\mathrm{ZrO}_{2}\right)_{x}\left(\mathrm{SiO}_{2}\right)_{1-x}$ compounds, and increasing the $\mathrm{ZrO}_{2}$ content resulted in larger convergence times. The reason for the slower convergence for these materials is the relatively complex structure of $\left(\mathrm{ZrO}_{2}\right)_{x}\left(\mathrm{SiO}_{2}\right)_{1-x}$ compounds, resulting in a larger variance in the total dipole moment and therefore requiring large times for the averaging to reach asymptotic values for the dielectric.

Figure 3 shows our results for five different glasses with variable $\mathrm{SiO}_{2}$ to $\mathrm{ZrO}_{2}$ ratios. First, we evaluated the method comparing the dielectric constant $\epsilon$ obtained from $\mathrm{MD}$ with the empirically known values for the end member oxides $\mathrm{SiO}_{2}$ and $\mathrm{ZrO}_{2}$. Our result for $\mathrm{SiO}_{2} \epsilon=3.75$ is in excellent agreement with the experimental range of 3.7-3.9, ${ }^{18,19}$ and for $\mathrm{ZrO}_{2}$ at $\epsilon \approx 22.5$, our value is within the experimental range $20-24 .^{20}$ Then, we computed the dielectric constant for four arbitrary $\left(\mathrm{ZrO}_{2}\right)_{x}\left(\mathrm{SiO}_{2}\right)_{1-x}$ compositions to showcase the ability to estimate their dielectric constants numerically. The dielectric constant increases with the $\mathrm{Zr}$ content from the value of pure $\mathrm{SiO}_{2}$ up to 15.6. An equivalent increase in the dielectric has been observed experimentally, ${ }^{16,21-23}$ yet our simulations overestimate the absolute value. Nevertheless, the qualitative increase with the $\mathrm{Zr}$ content is properly captured, which postulates the presented classical MD simulations as a predictive tool for the calculation of dielectric properties of highly disordered materials.

To explain the atomic scale origin of the $\epsilon$ increase with the $\mathrm{Zr}$ content, we explored two parameters that play a role in the dipole moment fluctuations within the classical atomic description adopted here, namely, the vibrations of the atomic positions and the atomic charges. The atomic positions are related to the vibrational frequencies of the material, and lower vibrational frequencies are associated with higher static dielectric constants. ${ }^{23}$ To study them, we have quantified the flexibility of the glass three-dimensional framework relying on the topological constraint theory (TCT). This approach has already been successful at describing a wide variety of glass properties, $^{14,15}$ including their dielectric response. ${ }^{25}$ TCT reduces the description of complex glass networks to a set of nodes (atoms) connected following a set of constraints defined by the chemical bonds. These constraints are divided in two sets: radial (or bond stretching, BS) constraints and angular (or bond bending, BB) constraints. Thus, the rigidity of the system is described by the average number of constraints per atom $n_{\mathcal{c}}$ where $n_{\mathrm{c}}=3$ sets the isostatic limit, $n_{\mathrm{c}}>3$ corresponds to a stressed-rigid domain, and $n_{\mathrm{c}}<3$ corresponds to flexible glasses.

Following the method proposed by Bauchy et al., ${ }^{26-28}$ the constraints are computed directly from the MD simulations by studying the pair distribution functions and partial bond angle distributions of each atomic species. The partial pair distribution
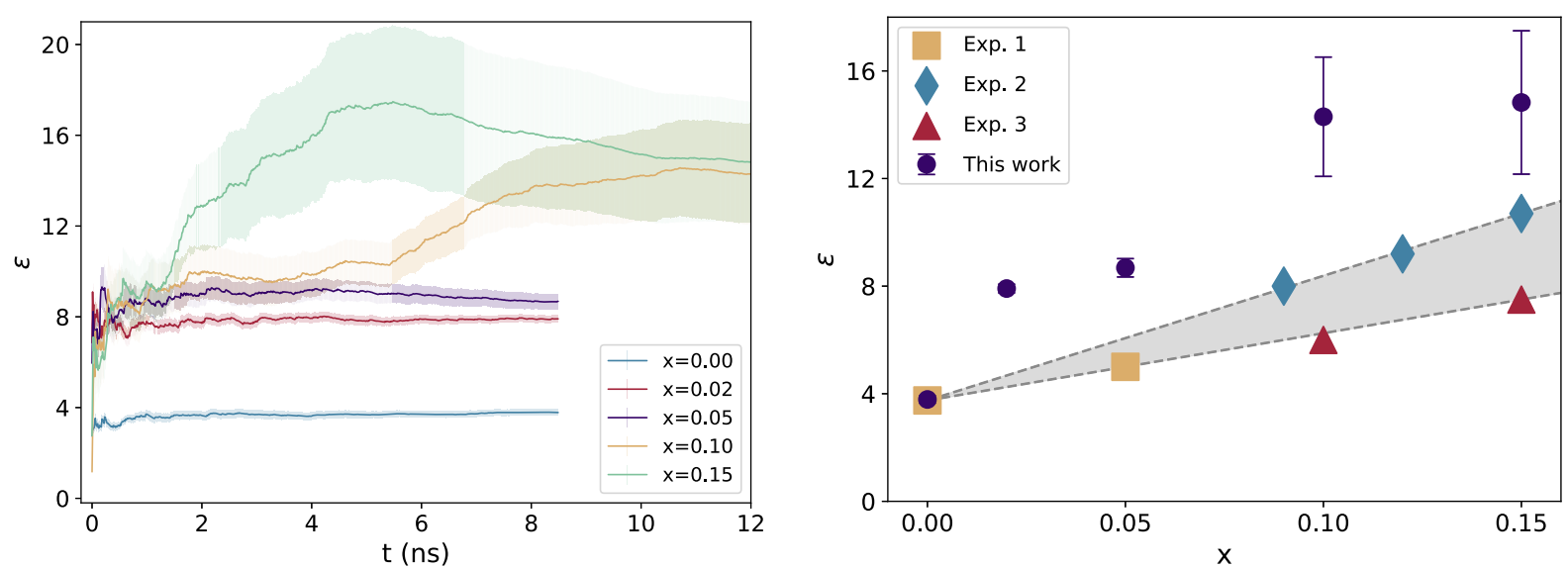

Figure 3. Left: Dielectric constant as a function of the simulation time for all studied systems. Right: Converged dielectric constants as a function of the composition along with experimental values from the literature. The experimental range is shadowed to guide the eye. ${ }^{21,23,24}$ 

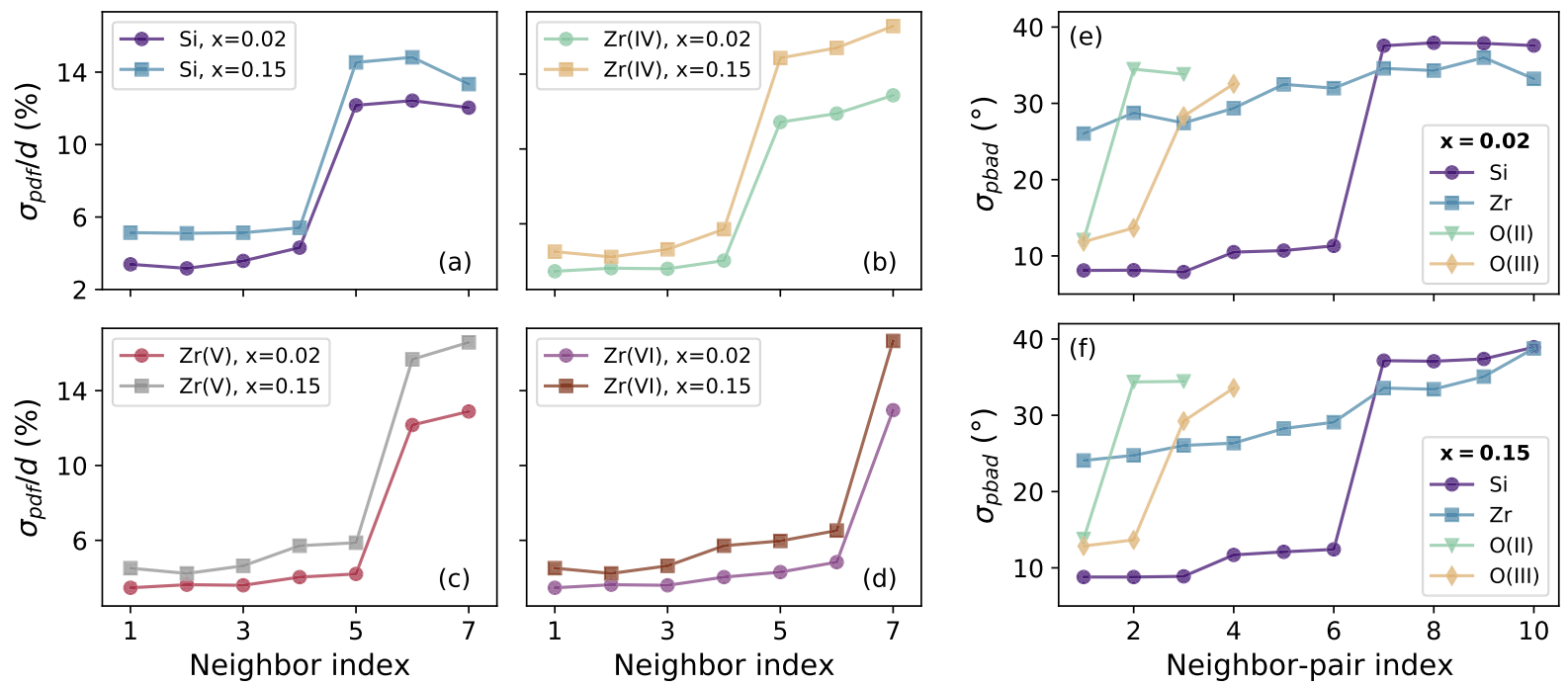

Figure 4. Standard deviation normalized by the bond length of the partial pair distribution functions for (a) $\mathrm{Si}$, (b) $\mathrm{Zr}(\mathrm{IV})$, (c) $\mathrm{Zr}(\mathrm{V})$, and (d) $\mathrm{Zr}(\mathrm{VI})$. Standard deviation of the $\mathrm{Si}-, \mathrm{Zr}-$, and $\mathrm{O}$-centered partial bond angle distributions for (e) $x=0.02$ and (f) $x=0.15$.

functions and bond angle distributions showed in Figure 1 allow to decide which radial and angular constraints are satisfied: a narrow distribution suggests an active constraint, whereas a broad one implies a broken constraint. Therefore, the standard deviation of each distribution must be computed. These are presented in Figure 4 for the radial and angular distributions. In order to do an appropriate counting, we studied independently the distributions of $\mathrm{Zr}$ according to their coordination, that is, $\mathrm{Zr}(\mathrm{IV}), \mathrm{Zr}(\mathrm{V})$, or $\mathrm{Zr}(\mathrm{VI})$, as well as $\mathrm{O}(\mathrm{II})$ and "tricluster" $\mathrm{O}$ (III). Since each $\mathrm{Si}$ or $\mathrm{Zr}$ atom is bonded to an oxygen atom, the oxygen atoms have been excluded from the radial constraint counting, assigning the full constraint to the cation. ${ }^{26-28}$ Considering all previous data, the radial standard deviations in Figure 4 show a clear gap between the deviations of the atoms within the first coordination shell and the second. Therefore, the number of BS constraints for each kind of atom is defined by its coordination: 4 constraints for $\mathrm{Si}$ and 4, 5, 6 for $\mathrm{Zr}(\mathrm{IV}), \mathrm{Zr}(\mathrm{V})$, and $\mathrm{Zr}(\mathrm{VI})$, respectively.

Regarding the angular constraints, the limit between broken and intact angular constraints is set around $\sigma=15 .^{27}$ As expected, O(II) has a single angular constraint, while "tricluster" $\mathrm{O}$ (III) has 2. Similarly, six angles around $\mathrm{Si}$ atoms are constrained, which correspond to the angles formed by its four neighbors. In this case, however, only five of these angles are independent, ${ }^{29}$ leading to five angular constraints. Finally, similar to $\mathrm{Al}$ in aluminosilicates, no angular constraint is assigned to $\mathrm{Zr}$ atoms due to the large variance of its partial angle distributions.

Once this analysis was done, we computed the average number of constraints per atom $n_{\mathrm{c}}$. It must be noted that the BS and $\mathrm{BB}$ constraints for each atomic species remain constant for the different compositions, and the total number of constraints per atom changes only due to the relative proportion of $\mathrm{O}$ (II)/ $\mathrm{O}$ (III) and the inclusion of more $\mathrm{Zr}$ without $\mathrm{BB}$ constraints. Denoting $z_{5}$ and $z_{6}$ as the fraction of $\mathrm{Zr}(\mathrm{V})$ and $\mathrm{Zr}(\mathrm{VI})$ with respect to the total $\mathrm{Zr}$, the average number of constraints is

$$
n_{\mathrm{c}}=\frac{11}{3}+x \frac{3 z_{5}+6 z_{6}-5}{3}
$$

Figure 5 depicts the relationship between the amount of $\mathrm{Zr}$ and the number of constraints. The number of constraints

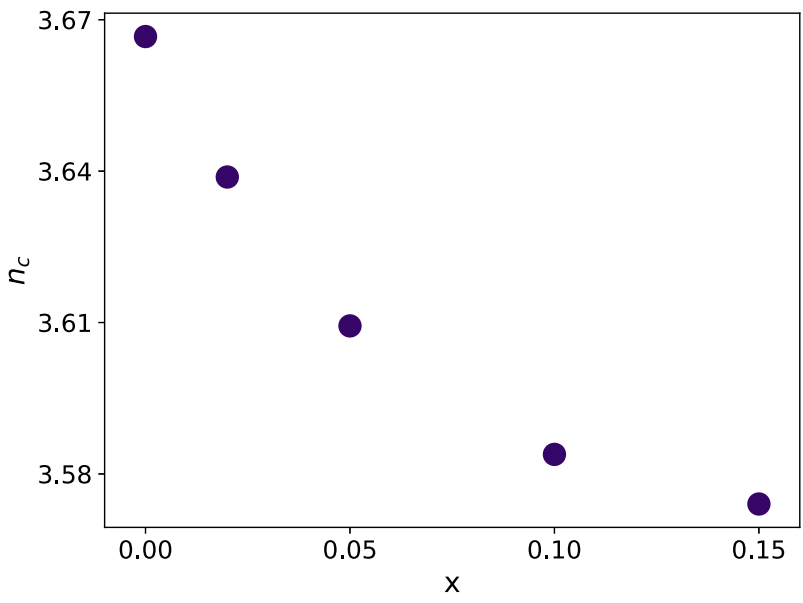

Figure 5. Average number of constraints per atom as a function of the $\mathrm{Zr}$ fraction.

decreases as more $\mathrm{Zr}$ is added, therefore becoming more flexible, which is consistent with the $\epsilon$ increase. As mentioned, the change of $n_{c}$ is due to the increase of $\mathrm{Zr}$ and $\mathrm{O}$ (III) in the structure. However, both species have a contrary effect on the flexibility: $\mathrm{Zr}$, without $\mathrm{BB}$ constraints, induces higher flexibility, while $\mathrm{O}$ (III) has an extra $\mathrm{BB}$ constraint compared to $\mathrm{O}$ (II), inducing rigidity. As a result, the mentioned increase of the flexibility is not very important, with $n_{\mathrm{c}}$ decreasing from 3.64 to 3.57. For comparison, the number of constraints may range from 2.8 to 3.5 when decreasing the $\mathrm{Ca} / \mathrm{Si}$ rate in $\mathrm{C}-\mathrm{S}-\mathrm{H}^{30}$ or from 2.9 to 3.4 for glassy aluminosilicates with $\mathrm{Al}$ molar fractions changing from 0 to $17 \%{ }^{14}$

We now turn to evaluate the atomic charges as a function of the $\mathrm{Zr}$ content. The charge distribution computed by the electronegativity equalization method (EEM) method $^{31}$ for the different atomic species is shown in Figure 6. The obtained charges are consistent with the expected values ${ }^{32}$ and with the phenomenological rule for the charge of mixed oxides proposed by Barr et al. ${ }^{33}$ They concluded from X-ray photoelectron spectroscopy experiments that in mixed oxides, the cation becomes more covalent than in the pure oxide and the cation becomes more ionic than in the pure oxide. Our simulation 
(a)

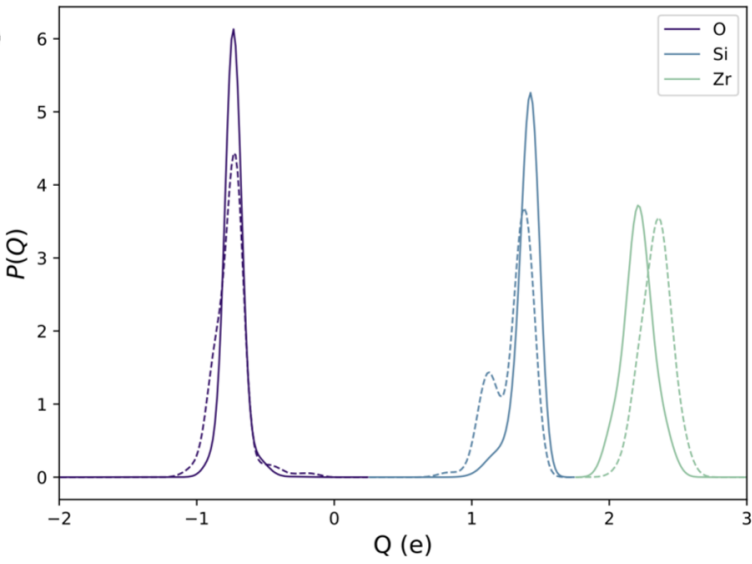

(b)

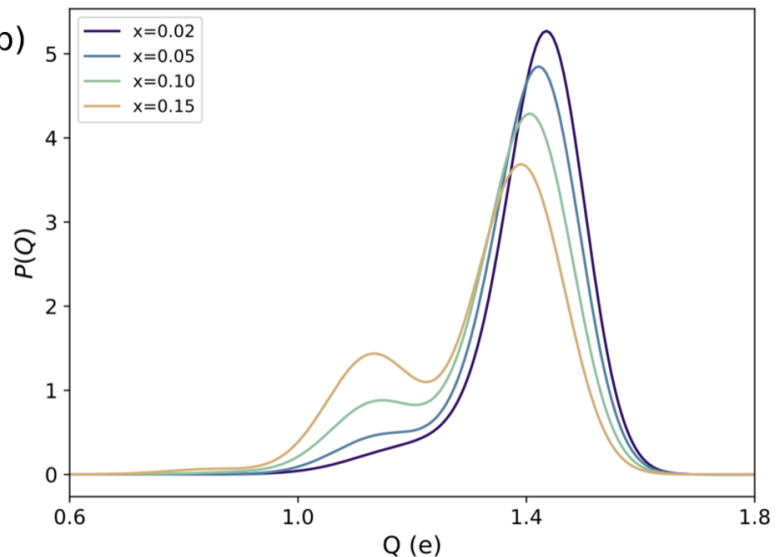

(c)

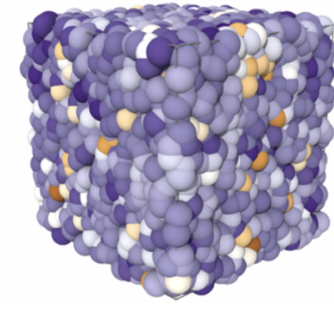

$x=0.05$

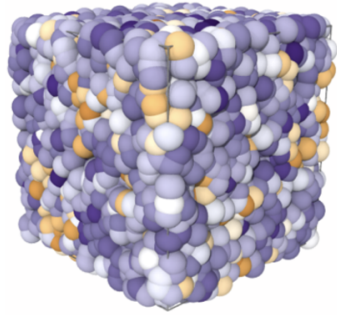

$x=0.10$

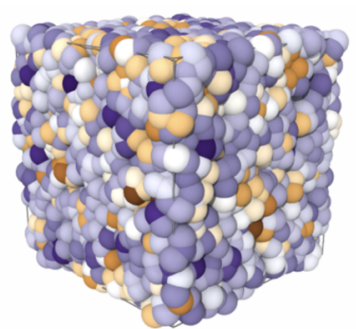

$x=0.15$

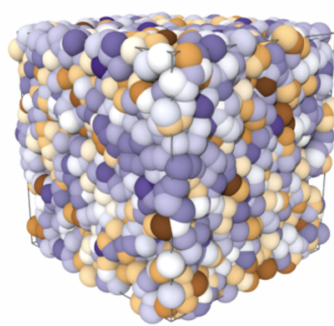

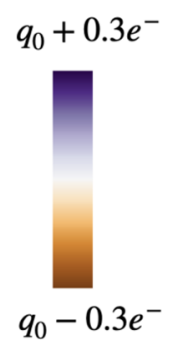

Figure 6. (a) $\mathrm{O}, \mathrm{Si}$, and $\mathrm{Zr}$ charge distributions. Solid lines are for $x=0.02$, and dashed lines are for 0.15 . (b) Si charge distributions for all structures. (c) Variation of the charges on the $\mathrm{Si}$ atoms around the central value defined by the local minimum between the two peaks shown in (b).

agrees with this empirical rule. From Figure 6, the charge distribution on $\mathrm{Zr}$ atoms shifts toward higher positive values at high $\mathrm{Zr}$ contents from $\approx 2.25$ to $\approx 2.5$, while the charge distribution of $\mathrm{Si}$ atoms shifts to lower positive values. The charges on oxygen atoms do not change substantially with the amount of $\mathrm{Zr}$, with a narrow distribution around $\approx-0.85$. The shift toward higher and lower charges for the two cations induces a larger heterogeneity in the local charge distribution that might increase the dipole moment fluctuations and consequently the static dielectric constant.

It is noteworthy that the charge decrease on $\mathrm{Si}$ atoms takes place by a shift in the intensity of two well-defined peaks. In Figure $6 \mathrm{c}$, we show the variation of charges on the $\mathrm{Si}$ atoms around the central value of $1.25 e^{-}$. Clearly, the population of the peaks is directly related to the amount of $\mathrm{Zr}$ in the system, and it is homogeneously distributed across the simulation box. However, we could not identify any relationship with the local chemical environment around each Si. Further research would be needed to understand these effects or identify if it could be a spurious result from the EEM method.

\section{CONCLUSIONS}

In this work, we reported a computer-aided characterization of dielectric materials of potential value for the CMOS technology. More specifically, we investigated $\mathrm{ZrO}_{2}$ and $\left(\mathrm{ZrO}_{2}\right)_{x}\left(\mathrm{SiO}_{2}\right)_{1-x}$ glasses with variable $\mathrm{Zr}$ contents using classical MD with ReaxFF empirical potentials.

First, we merged two ReaxFF sets $(\mathrm{Si} / \mathrm{O} / \mathrm{H}$ and $\mathrm{Zr} / \mathrm{O} / \mathrm{H})$ and parameterized non available cross terms with a home-made code to build a new $\mathrm{Si} / \mathrm{O} / \mathrm{Zr} / \mathrm{H}$ set. Then, we prepared $\left(\mathrm{ZrO}_{2}\right)_{x}\left(\mathrm{SiO}_{2}\right)_{1-x}$ glasses and computed the dielectric constant from the average dipole moment fluctuations of the simulation box. We find results in qualitative agreement with the available experimental data: as the $\mathrm{Zr}$ content increases, the dielectric constant of the amorphous $\left(\mathrm{ZrO}_{2}\right)_{x}\left(\mathrm{SiO}_{2}\right)_{1-x}$ steadily increases. Analyzing our simulations, we can attribute such an increase to two factors. On the one hand, there is an increase of the framework flexibility as the $\mathrm{Zr}$ content increases, that we have quantified from the point of view of the TCT. On the other hand, there is an increasing heterogeneity of the charge in the cations, with $\mathrm{Zr}$ becoming more ionic and Si more covalent as the $\mathrm{Zr}$ content increases. Those two factors, from which the latter is the more marked, induce larger fluctuations in the dipole moment and, consequently, a larger static dielectric constant.

To the best of our knowledge, our work is the first reported use of classical MD simulations for predicting the dielectric constant of amorphous solids. Our work and findings illustrate an example of how MD-based CADM can constitute an effective and attractive methodology for advancing complex CMOS technology which is highly dependent on the intricate interaction of a large number of well-known and ill-studied materials.

\section{METHODS}

Ab Initio Simulations. We used density functional theory (DFT) to perform calculations of small clusters containing $\mathrm{Si}, \mathrm{O}$, $\mathrm{Zr}$, and $\mathrm{H}$. The Gaussian-16 program $^{34}$ was used to perform these calculations. In order to select an accurate exchangecorrelation $(\mathrm{XC})$ functional to compute structures and energies, we first compared results from different XC functionals with those from the MP2 perturbation theory. ${ }^{35,36}$ We ended up using the B3LYP functional with the D3 version of Grimme's dispersion. ${ }^{37}$ Equilibrium $\mathrm{Zr}-\mathrm{O}-\mathrm{Si}$ angles obtained using B3LYP were equal to those calculated using MP2, providing evidence of the accurate predictive capability of this functional for our application. $\mathrm{Si}, \mathrm{O}$, and $\mathrm{H}$ were described at the full 
electron level by a variation of the $6-31 \mathrm{G}$ basis set with single diffuse functions as well as $d$ functions. ${ }^{38,39} \mathrm{Zr}$ was described using an energy-consistent relativistic pseudo-potential along with the correlation consistent basis set with diffuse functions. ${ }^{40}$ This pseudo-potential/basis set pair is specific for $4 \mathrm{~d}$ transition metals and is available through the basis exchange database $e^{41-43}$ under aug-cc-pVTZ-PP.

In the end, we calculated the electronic energy for two systems subjected to variations: one for a system with $\mathrm{Zr}-\mathrm{O}-\mathrm{Si}$ angles varied across a range of values away from the equilibrium angle and a second system with hydrogen bonds also varied over a range of values away from the equilibrium bond length. More details on the molecular systems and $a b$ initio simulations are provided in the Supporting Information document. These two atomic structures and their corresponding energies formed the basis for the next step.

ReaxFF Parametrization. We selected ReaxFF as our choice of classical forcefield because of its broad applicability. For the specific problem of dielectric constants, ReaxFF is suitable due to the $\mathrm{EEM}^{31}$ that let us compute dipole moment fluctuations due not only to atomic displacements but also to charge fluctuations. ReaxFF was initially introduced to study hydrocarbon systems, ${ }^{11}$ yet ReaxFF parameter sets were later developed and used to successfully describe various properties of semiconductors and transition metals as well as various processes related to those systems. ${ }^{10,44,45}$

The ReaxFF parameters for the $\mathrm{Si} / \mathrm{Zr} / \mathrm{O} / \mathrm{H}$ set we parameterized are based on the independent sets made by Fogarty et al. for $\mathrm{SiO}_{2}{ }^{44}$ and van Duin et al. for $\mathrm{ZrO}_{2} \cdot{ }^{45}$ Since electropositive elements such as $\mathrm{Hf}$ and $\mathrm{Zr}$ tend to form bonds with oxygen rather than $\mathrm{Si}^{46}$ parameterizing the $\mathrm{Zr}-\mathrm{O}-$ $\mathrm{Si}$ angular terms seems sufficient to accurately model both bulk and interface cases with $\left(\mathrm{ZrO}_{2}\right)_{x}\left(\mathrm{SiO}_{2}\right)_{1-x}$, at least for low concentrations, when $\mathrm{Zr}$ acts a network modifier.

We optimized our ReaxFF parameters using the molecular system calculated by DFT. The optimization criterion was to minimize the mean-square error of the energy computed using our forcefield versus the energy determined using DFT. We developed an optimization tool specifically for this work. The python code, named Pyfield, is provided in GitHub. ${ }^{47}$ Pyfield uses simulated annealing and a genetic algorithm as its main optimization engine. Additional details on Pyfield are also provided in the Supporting Information.

Preparation of $\left(\mathrm{ZrO}_{2}\right)_{x}\left(\mathrm{SiO}_{2}\right)_{1-x}$ Amorphous Systems. Using the optimized forcefield produced by our Pyfield tool, we performed MD simulations using LAMMPS ${ }^{48}$ (2018 release). The following steps were taken to prepare the system:

- Placement: The systems we studied contain 24,000 atoms, 2 orders of magnitude larger than previous attempts to compute static dielectric constants with DFT methods, which lead to a better sampling of the properties. The amorphous $\left(\mathrm{ZrO}_{2}\right)_{x}\left(\mathrm{SiO}_{2}\right)_{1-x}$ systems were prepared based on a technique previously used ${ }^{44,49}$ by randomly placing $8000 \mathrm{SiO}_{2}$ molecules in a box with the dimension of $72 \times 70 \times 72 \AA^{3}$ and randomly replacing a portion of $\mathrm{Si}$ atoms with $\mathrm{Zr}$ to achieve the desired ratio. We then proceeded with annealing the system as per the following step.

- Annealing: We annealed the system using an NPT ensemble by initiating it at $3000 \mathrm{~K}$ and cooling it down to $300 \mathrm{~K}$ over a span of $1 \mathrm{~ns}$. We then equilibrated the system at $300 \mathrm{~K}$ for another $100 \mathrm{ps}$.
For our NVT and NPT simulations, we used a Nose-Hoover thermostat and barostat. ${ }^{50,51}$ The integration time steps we used for the Velocity-Verlet algorithm were set to 0.1 fs for the above steps (Figure 7).
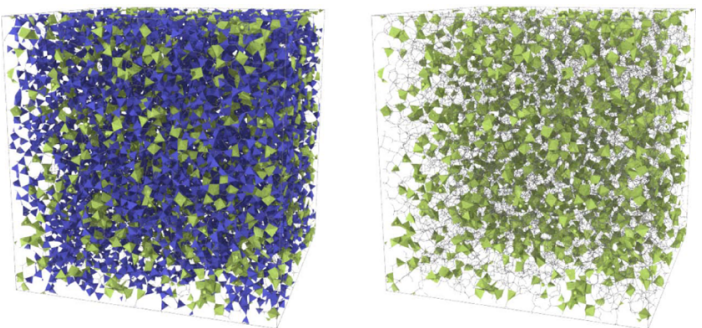

Figure 7. Left: Representation of the amorphous $\left(\mathrm{ZrO}_{2}\right)_{0.15}\left(\mathrm{SiO}_{2}\right)_{0.85}$ atomic structure. Blue and green coordination polyhedra represent the silicate and zirconate groups, respectively. Right: Detailed view of the zirconate polyhedra for the same sample.

Material Characterization. The ultimate objective of our work was to determine the permittivity of $\mathrm{Zr}$-doped silica glasses which we assumed to be homogeneous and isotropic. The standard definition of polarization $\vec{P}$ is such that $\vec{P}=\epsilon_{0} \chi \vec{E}$, where $\vec{E}$ is the applied electric field, $\epsilon_{0}$ is the vacuum permittivity, and $\chi$ is the dispersion. Ideally, we would want to apply an electric field, collect the polarization data, and compute $\chi$ which would lead to the estimation of the relative permittivity $\epsilon=\chi+1$. However, applying an electric field to a classical MD simulation is not trivial. Instead, a reasonable approach is to use dipole fluctuations to calculate bulk $\left(\mathrm{ZrO}_{2}\right)_{x}\left(\mathrm{SiO}_{2}\right)_{1-x}$ permittivity in a fashion similar to how various liquids have been studied ${ }^{52-54}$ using the expression

$$
\epsilon=1+\frac{\left\langle\left(M^{E=0}\right)^{2}\right\rangle-\left\langle M^{E=0}\right\rangle^{2}}{3 k_{\mathrm{b}} T V \epsilon_{0}}
$$

where the $\left\langle\left(M^{E=0}\right)^{2}\right\rangle-\left\langle M^{E=0}\right\rangle^{2}$ term denotes the mean-square deviation of the total dipole moment, $k_{\mathrm{b}}$ is Boltzmann's constant, $T$ is the temperature, and $V$ is the volume over which the computation is performed. To compute the ensemble averages $\left(\left\langle\left(M^{E=0}\right)^{2}\right\rangle\right.$ and $\left.\left\langle M^{E=0}\right\rangle\right)$, we performed the simulations based on the following procedure:

- Initiation: Systems are initiated and equilibrated at $300 \mathrm{~K}$ for 200,000 steps where each time step is $1 \mathrm{fs}$.

- Fluctuation: Systems were allowed to fluctuate using an NVT ensemble at $300 \mathrm{~K}$ over a span of 8 to $12 \mathrm{~ns}$ depending on the sample with a time step of $1 \mathrm{fs}$.

The total dipole moment of the system was computed during the fluctuation step at every $20 \mathrm{ps}$, and we used them to compute the relative permittivity as a function of time. The dipole moments are computed according to $M=\sum_{i} q_{i} \vec{r}_{i}$ where $q_{i}$ is the partial charge of a particle and $\vec{r}_{i}$ is the location vector of the charge from an arbitrary origin.

\section{APPENDIX}

In this section, we provide a simple derivation of eq 2 . The derivation should help the readers understand the limits of its applicability. Assuming an electric field in the $\vec{x}$ direction

$$
\vec{P}_{x}=\frac{\left\langle\left\langle M_{x}^{E=E_{x}}-\left\langle M_{x}^{E=0}\right\rangle\right\rangle\right.}{V}
$$


where \langle\rangle stands for ensemble average, $M_{x}$ is the dipole moment in the $\vec{x}$ direction, and $V$ is the system's volume. Using the Boltzmann law, we have

$$
\begin{array}{r}
\left\langle\left\langle M_{x}^{E=E_{x}}-\left\langle M_{x}^{E=0}\right\rangle\right\rangle\right. \\
=\frac{\int \ldots \int d_{p_{1}} \ldots d_{p_{N}}\left(M_{x}^{E=E_{x}}-\left\langle M_{x}^{E=0}\right\rangle\right) \mathrm{e}^{-\left(U-M_{x}^{E=E_{x}}\right) / K T}}{\int \ldots \int d_{p_{1}} \ldots d_{p_{N}} \mathrm{e}^{-\left(U-M_{x}^{\left.E=E_{x}\right) / K T}\right.}}
\end{array}
$$

where the integration is over all degrees of freedom of all of the particles and $U$ is the energy of the system when no electric field is applied. Performing a Taylor series expansion of eq 4 at $M_{x}^{E=0}$ and only keeping the first-order term yields

$$
\begin{aligned}
& \left\langle M_{x}^{E=E_{x}}-\left\langle M_{x}^{E=0}\right\rangle\right\rangle=\frac{\partial}{\partial E}\left\{\int \ldots \int d_{p_{1}} \ldots d_{p_{N}}\left(M_{x}^{E=E_{x}}-\left\langle M_{x}^{E=0}\right\rangle\right)\right. \\
& \left.\exp \left[-\left(U-M_{x}^{E=E_{x}}\right) / K T\right]\right\} /\left\{\int \ldots \int d_{p_{1}} \ldots d_{p_{N}}\right. \\
& \left.\exp \left[-\left(U-M_{x}^{E=E_{x}}\right) / K T\right]\right\}\left.\right|_{E=E_{x}} \\
& \times E_{x}=\left\{\int \ldots \int d_{p_{1}} \ldots d_{p_{N}}\left(\left(M_{x}^{E=E_{x}}\right)^{2}-M_{x}^{E=E_{x}} \times\left\langle M_{x}^{E=0}\right\rangle\right)\right. \\
& \left.\exp \left[-\left(U-M_{x}^{E=E_{x}}\right) / K T\right]\right\} /\left\{K T \times \int \ldots \int d_{p_{1}} \ldots d_{p_{N}}\right. \\
& \left.\exp \left[-\left(U-M_{x}^{E=E_{x}}\right) / K T\right]\right\}\left.\right|_{E=E_{x}} \\
& \times E_{x}=\frac{\left\langle\left(M_{x}^{E=0}\right)^{2}\right\rangle-\left\langle M_{x}^{E=0}\right\rangle^{2}}{K T} \times E_{x}=\frac{\left\langle\left(M^{E=0}\right)^{2}\right\rangle-\left\langle M^{E=0}\right\rangle^{2}}{3 K T} \times E_{x}
\end{aligned}
$$

where the last equality is valid if the material is isotropic. ${ }^{a}$ By substituting the right-hand side of eq 5 with $\left\langle\left\langle M_{x}^{E=E_{x}}-\left\langle M_{x}^{E=0}\right\rangle\right\rangle\right.$ in eq 3 and considering that $\vec{P}_{x}=\epsilon 0 \chi \vec{E}_{x}$, we have

$$
\chi=\frac{\left\langle\left(M^{E=0}\right)^{2}\right\rangle-\left\langle M^{E=0}\right\rangle^{2}}{3 K T V \epsilon_{0}}
$$

and $\epsilon=\chi+1$.

\section{ASSOCIATED CONTENT}

\section{(5) Supporting Information}

The Supporting Information is available free of charge at https://pubs.acs.org/doi/10.1021/acsomega.1c02533.

Complete details of DFT and MD simulations along with the forcefield (PDF)

\section{AUTHOR INFORMATION}

\section{Corresponding Authors}

S. Arash Sheikholeslam - Department of Electrical and Computer Engineering, UBC, V6T1Z4 Vancouver, Canada; (1) orcid.org/0000-0001-6918-6008; Email: sarashs@ ece.ubc.ca

Jon López-Zorrilla - Department of Physics, University of the Basque Country UPV/EHU, 48330 Leioa, Bizkaia, Spain; Email: jon.lopezz@ehu.eus

Hegoi Manzano - Department of Physics, University of the Basque Country UPV/EHU, 48330 Leioa, Bizkaia, Spain; (1) orcid.org/0000-0001-7992-2718;

Email: hegoi.manzano@ehu.eus

Saamaan Pourtavakoli - Eigen Research Inc., M5G1L5 Toronto, Canada; Email: saamaan@eigenresearch.ca
André Ivanov - Department of Electrical and Computer Engineering, UBC, V6T1Z4 Vancouver, Canada;

Email: ivanov@ece.ubc.ca

Complete contact information is available at:

https://pubs.acs.org/10.1021/acsomega.1c02533

\section{Notes}

The authors declare no competing financial interest.

\section{ACKNOWLEDGMENTS}

This research was enabled in part by support provided by Compute Canada (www.computecanada.ca). Computations were performed on the Niagara supercomputer at the SciNet HPC Consortium. SciNet is funded by the Canada Foundation for Innovation, the Government of Ontario, Ontario Research Fund-Research Excellence, and the University of Toronto.

\section{ADDITIONAL NOTE}

${ }^{a}$ We have $\langle M\rangle / 3=\left\langle M_{x}\right\rangle=\left\langle M_{y}\right\rangle=\left\langle M_{z}\right\rangle$ due to isotropy. Also, $M$ $=M_{x}+M_{y}+M_{z}$ and $M^{2}=M_{x}^{2}+M_{y}^{2}+M_{z}^{2}$. This last one is true in general.

\section{REFERENCES}

(1) Darbandy, G.; Lime, F.; Cerdeira, A.; Estrada, M.; Garduño, I.; Iñiguez, B. Study of potential high- $\mathrm{k}$ dielectric for UTB SOI MOSFETs using analytical modeling of the gate tunneling leakage. Semicond. Sci. Technol. 2011, 26, 115002

(2) Wilson, L. International technology roadmap for semiconductors (ITRS); Semiconductor Industry Association, 2013.

(3) Salaün, A.; Grampeix, H.; Buckley, J.; Mannequin, C.; Vallée, C.; Gonon, P.; Jeannot, S.; Gaumer, C.; Gros-Jean, M.; Jousseaume, V. Investigation of $\mathrm{HfO}_{2}$ and $\mathrm{ZrO}_{2}$ for Resistive Random Access Memory applications. Thin Solid Films 2012, 525, 20-27.

(4) Panda, D.; Tseng, T.-Y. Growth, dielectric properties, and memory device applications of $\mathrm{ZrO}_{2}$ thin films. Thin Solid Films 2013, 531, 120 .

(5) Ye, G.; Wang, H.; Arulkumaran, S.; Ng, G. I.; Hofstetter, R.; Li, Y.; Anand, M. J.; Ang, K. S.; Maung, Y. K. T.; Foo, S. C. Atomic layer deposition of $\mathrm{ZrO} 2$ as gate dielectrics for $\mathrm{AlGaN} / \mathrm{GaN}$ metal-insulatorsemiconductor high electron mobility transistors on silicon. Appl. Phys. Lett. 2013, 103, 142109.

(6) Moghadam, P. Z.; Islamoglu, T.; Goswami, S.; Exley, J.; Fantham, M.; Kaminski, C. F.; Snurr, R. Q.; Farha, O. K.; Fairen-Jimenez, D. Computer-aided discovery of a metal-organic framework with superior oxygen uptake. Nat. Commun. 2018, 9, 1378.

(7) Pettifor, D. G. Computer-aided materials design: bridging the gaps between physics, chemistry and engineering. Phys. Educ. 1997, 32, 164.

(8) Siu, S. W. I.; Pluhackova, K.; Böckmann, R. A. Optimization of the OPLS-AA force field for long hydrocarbons. J. Chem. Theory Comput. 2012, 8, 1459-1470

(9) Jaramillo-Botero, A.; Naserifar, S.; Goddard, W. A., III General multiobjective force field optimization framework, with application to reactive force fields for silicon carbide. J. Chem. Theory Comput. 2014, $10,1426-1439$.

(10) Chenoweth, K.; Cheung, S.; van Duin, A. C. T.; Goddard, W. A., III; Kober, E. M. Simulations on the thermal decomposition of a poly (dimethylsiloxane) polymer using the ReaxFF reactive force field. J. Am. Chem. Soc. 2005, 127, 7192-7202.

(11) Van Duin, A. C. T.; Dasgupta, S.; Lorant, F.; Goddard, W. A. ReaxFF: a reactive force field for hydrocarbons. J. Phys. Chem. A 2001, 105, 9396-9409.

(12) Senftle, T. P.; Hong, S.; Islam, M. M.; Kylasa, S. B.; Zheng, Y.; Shin, Y. K.; Junkermeier, C.; Engel-Herbert, R.; Janik, M. J.; Aktulga, H. M.; et al. The ReaxFF reactive force-field: development, applications and future directions. npj Comput. Mater. 2016, 2, 15011. 
(13) Sokolov, V. O.; Sulimov, V. B. Threefold coordinated oxygen atom in silica glass. J. Non-Cryst. Solids 1997, 217, 167-172.

(14) Oey, T.; La Plante, E. C.; Falzone, G.; Yang, K.; Wada, A.; Bauchy, M.; Bullard, J. W.; Sant, G. Topological controls on aluminosilicate glass dissolution: Complexities induced in hyperalkaline aqueous environments. J. Am. Ceram. Soc. 2020, 103, 6198-6207.

(15) Oey, T.; Kumar, A.; Pignatelli, I.; Yu, Y.; Neithalath, N.; Bullard, J. W.; Bauchy, M.; Sant, G. Topological controls on the dissolution kinetics of glassy aluminosilicates. J. Am. Ceram. Soc. 2017, 100, 55215527.

(16) Lucovsky, G.; Rayner, G. B., Jr. Microscopic model for enhanced dielectric constants in low concentration $\mathrm{ZrO}_{2}$-rich noncrystalline $\mathrm{Zr}$ and Hf silicate alloys. Appl. Phys. Lett. 2000, 77, 2912-2914.

(17) Devanathan, R.; Corrales, L. R.; Weber, W. J.; Chartier, A.; Meis, C. Molecular dynamics simulation of disordered zircon. Phys. Rev. B: Condens. Matter Mater. Phys. 2004, 69, 064115.

(18) Moon, H.; Cho, S. K.; Garrell, R. L.; Kim, C.-J. C. Low voltage electrowetting-on-dielectric. J. Appl. Phys. 2002, 92, 4080-4087.

(19) DiMaria, D. J.; Stasiak, J. W. Trap creation in silicon dioxide produced by hot electrons. J. Appl. Phys. 1989, 65, 2342-2356.

(20) Kukli, K.; Forsgren, K.; Ritala, M.; Leskelä, M.; Aarik, J.; Hårsta, A. Dielectric properties of zirconium oxide grown by atomic layer deposition from iodide precursor. J. Electrochem. Soc. 2001, 148, F227F232.

(21) Ferrer, F. J.; Frutos, F.; García-López, J.; Jiménez, C.; Yubero, F. Electrical characteristics of mixed $\mathrm{Zr}-\mathrm{Si}$ oxide thin films prepared by ion beam induced chemical vapor deposition at room temperature. Thin Solid Films 2009, 517, 5446-5452.

(22) Naoi, T. A.; Paik, H.; Green, M. L.; van Dover, R. B. Dielectric properties of amorphous $\mathrm{Zr}-\mathrm{Al}-\mathrm{O}$ and $\mathrm{Zr}-\mathrm{Si}-\mathrm{O}$ thin films. J. Adv. Dielectr. 2015, 05, 1550010.

(23) Rignanese, G.-M.; Detraux, F.; Gonze, X.; Bongiorno, A.; Pasquarello, A. Dielectric constants of $\mathrm{Zr}$ silicates: a first-principles study. Phys. Rev. Lett. 2002, 89, 117601.

(24) Ouyang, L.; Ching, W. Y. Electronic structure and dielectric properties of dielectric gate material $\left(\mathrm{ZrO}_{2}\right) x\left(\mathrm{SiO}_{2}\right)_{1} \_x$. J. Appl. Phys. 2004, 95, 7918-7924.

(25) Micoulaut, M. Concepts and applications of rigidity in noncrystalline solids: a review on new developments and directions. Adv. Phys.: X 2016, 1, 147-175.

(26) Bauchy, M.; Micoulaut, M. Atomic scale foundation of temperature-dependent bonding constraints in network glasses and liquids. J. Non-Cryst. Solids 2011, 357, 2530-2537.

(27) Bauchy, M.; Abdolhosseini Qomi, M. J.; Bichara, C.; Ulm, F.-J.; Pellenq, R. J.-M. Nanoscale structure of cement: viewpoint of rigidity theory. J. Phys. Chem. C 2014, 118, 12485-12493.

(28) Bauchy, M.; Wang, M.; Yu, Y.; Wang, B.; Krishnan, N. M. A.; Masoero, E.; Ulm, F.-J.; Pellenq, R. Topological Control on the Structural Relaxation of Atomic Networks under Stress. Phys. Rev. Lett. 2017, 119, 035502.

(29) Thorpe, M. F. Continuous deformations in random networks. J. Non-Cryst. Solids 1983, 57, 355-370.

(30) Bauchy, M.; Qomi, M. J. A.; Bichara, C.; Ulm, F.-J.; Pellenq, R. J.M. Rigidity Transition in Materials: Hardness is Driven by Weak Atomic Constraints. Phys. Rev. Lett. 2015, 114, 125502.

(31) Mortier, W. J.; Ghosh, S. K.; Shankar, S. Electronegativityequalization method for the calculation of atomic charges in molecules. J. Am. Chem. Soc. 1986, 108, 4315-4320.

(32) Guittet, M. J.; Crocombette, J. P.; Gautier-Soyer, M. Bonding and XPS chemical shifts in $\mathrm{ZrSiO}_{4}$ versus $\mathrm{SiO}_{2}$ and $\mathrm{ZrO}_{2}$ : Charge transfer and electrostatic effects. Phys. Rev. B: Condens. Matter Mater. Phys. 2001, 63, 125117.

(33) Barr, T. L. Recent advances in x-ray photoelectron spectroscopy studies of oxides. J. Vac. Sci. Technol., A 1991, 9, 1793-1805.

(34) Frisch, M. J.; et al. Gaussian 16, revision a. 03. 2016; Gaussian Inc.: Wallingford CT, 2016; Vol. 2.

(35) Frisch, M. J.; Head-Gordon, M.; Pople, J. A. Semi-direct algorithms for the MP2 energy and gradient. Chem. Phys. Lett. 1990, $166,281-289$.
(36) Frisch, M. J.; Head-Gordon, M.; Pople, J. A. A direct MP2 gradient method. Chem. Phys. Lett. 1990, 166, 275-280.

(37) Grimme, S.; Antony, J.; Ehrlich, S.; Krieg, H. A consistent and accurate $\mathrm{ab}$ initio parametrization of density functional dispersion correction (DFT-D) for the 94 elements H-Pu. J. Chem. Phys. 2010, 132, 154104.

(38) Petersson, G. A.; Bennett, A.; Tensfeldt, T. G.; Al-Laham, M. A.; Shirley, W. A.; Mantzaris, J. A complete basis set model chemistry. I. The total energies of closed shell atoms and hydrides of the first row elements. J. Chem. Phys. 1988, 89, 2193-2218.

(39) Petersson, G. A.; Al-Laham, M. A. A complete basis set model chemistry. II. Open shell systems and the total energies of the first row atoms. J. Chem. Phys. 1991, 94, 6081-6090.

(40) Peterson, K. A.; Figgen, D.; Dolg, M.; Stoll, H. Energy-consistent relativistic pseudopotentials and correlation consistent basis sets for the 4d elements Y-Pd. J. Chem. Phys. 2007, 126, 124101.

(41) Pritchard, B. P.; Altarawy, D.; Didier, B.; Gibson, T. D.; Windus, T. L. New basis set exchange: An open, up-to-date resource for the molecular sciences community. J. Chem. Inf. Model. 2019, 59, 48144820.

(42) Feller, D. The role of databases in support of computational chemistry calculations. J. Comput. Chem. 1996, 17, 1571-1586.

(43) Schuchardt, K. L.; Didier, B. T.; Elsethagen, T.; Sun, L.; Gurumoorthi, V.; Chase, J.; Li, J.; Windus, T. L. Basis set exchange: a community database for computational sciences. J. Chem. Inf. Model. 2007, 47, 1045-1052.

(44) Fogarty, J. C.; Aktulga, H. M.; Grama, A. Y.; Van Duin, A. C. T.; Pandit, S. A. A reactive molecular dynamics simulation of the silicawater interface. J. Chem. Phys. 2010, 132, 174704.

(45) van Duin, A. C. T.; Merinov, B. V.; Han, S. S.; Dorso, C. O.; Goddard, W. A. ReaxFF Reactive Force Field for the Y-Doped $\mathrm{BaZrO}_{3}$ Proton Conductor with Applications to Diffusion Rates for Multigranular Systems. J. Phys. Chem. A 2008, 112, 11414-11422.

(46) Peacock, P. W.; Robertson, J. Bonding, Energies, and Band Offsets of $\mathrm{Si}-\mathrm{ZrO}_{2}$ and $\mathrm{HfO}_{2}$ Gate Oxide Interfaces. Phys. Rev. Lett. 2004, 92, 057601.

(47) Sheikholeslam, S. A. Pyfield. https://github.com/sarashs/ Python-forcefield-optimizer, 2020.

(48) Plimpton, S. Fast parallel algorithms for short-range molecular dynamics. J. Comput. Phys. 1995, 117, 1-19.

(49) Sheikholeslam, S. A.; Manzano, H.; Grecu, C.; Ivanov, A. Reduced hydrogen diffusion in strained amorphous $\mathrm{SiO} 2$ : understanding ageing in MOSFET devices. J. Mater. Chem. C 2016, 4, 81048110.

(50) Hoover, W. G. Canonical dynamics: Equilibrium phase-space distributions. Phys. Rev. A: At., Mol., Opt. Phys. 1985, 31, 1695-1697.

(51) Nosé, S. A unified formulation of the constant temperature molecular dynamics methods. J. Chem. Phys. 1984, 81, 511-519.

(52) Raabe, G.; Sadus, R. J. Molecular dynamics simulation of the dielectric constant of water: The effect of bond flexibility. J. Chem. Phys. 2011, 134, 234501.

(53) Olmi, R.; Bittelli, M. Can molecular dynamics help in understanding dielectric phenomena? Meas. Sci. Technol. 2016, 28, 014003.

(54) Neumann, M. Dipole moment fluctuation formulas in computer simulations of polar systems. Mol. Phys. 1983, 50, 841-858. 